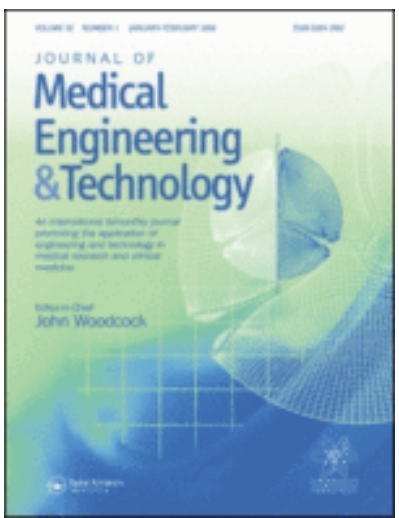

\title{
Variability in body temperature in healthy adults and in patients receiving chemotherapy: prospective observational cohort study
}

\begin{tabular}{|r|l|}
\hline Journal: & Journal of Medical Engineering \& Technology \\
\hline Manuscript ID & TMET-2019-0027 \\
\hline Danuscript Type: & Innovation \\
\hline Author: & $10-$ Mar-2019 \\
\hline Komplete List of Authors: & $\begin{array}{l}\text { Frazer, John; University of Oxford Medical Sciences Division } \\
\text { Barnes, Grace; University of Oxford Medical Sciences Division } \\
\text { Woodcock, Victoria; Weatherall Institute of Molecular Medicine } \\
\text { Department of Oncology } \\
\text { Flannigan, Eliz; Churchill Hospital, Clinical Haematology and Oncology } \\
\text { Littlewood, Tim; University of Oxford Medical Sciences Division } \\
\text { Stevens, Richard; University of Oxford Department of Primary Health } \\
\text { Care } \\
\text { Fleming, Susannah; University of Oxford Department of Primary Health } \\
\text { Care } \\
\text { Ashdown, Helen; University of Oxford Department of Primary Health } \\
\text { Care }\end{array}$ \\
\hline Keywords: & $\begin{array}{l}\text { Temperature variability, Chemotherapy, Neutropenic sepsis, } \\
\text { Thermometry }\end{array}$ \\
\hline &
\end{tabular}

\section{SCHOLARONE" \\ Manuscripts}




\section{Variability in body temperature in healthy adults and in patients receiving chemotherapy: prospective observational cohort study}

Frazer JS ${ }^{\mathrm{a}}$, Barnes $\mathrm{G}^{\mathrm{a}}$, Woodcock V ${ }^{\mathrm{b}}$, Flanagan $\mathrm{E}^{\mathrm{b}}$, Littlewood T $\mathrm{T}^{\mathrm{a}, \mathrm{b}}$, Stevens RJc, Fleming $\mathrm{S}^{\mathrm{c}}$, Ashdown $\mathrm{HF}^{\mathrm{c}}$

${ }^{a}$ Medical School, University of Oxford; ${ }^{b}$ Oxford University Hospitals NHS Trust; ${ }^{c}$ Nuffield Department of Primary Care Health Sciences, University of Oxford

Corresponding author: helen.ashdown@phc.ox.ac.uk

Word count: 4,873 words (excluding abstract, figures, and references). 


\title{
Variability in body temperature in healthy adults and in patients receiving chemotherapy: prospective observational cohort study
}

\begin{abstract}
Between-individual variability of body temperature has been little investigated, but is of clinical importance: for example, in detection of neutropenic sepsis during chemotherapy. We studied within-person and between-person variability in temperature in healthy adults and those receiving chemotherapy using a prospective observational design involving 29 healthy participants and 23 patients undergoing chemotherapy. Primary outcome was oral temperature.
\end{abstract} We calculated each patient's mean temperature, standard deviation within each patient (withinperson variability), and between patients (between-person variability). Secondary analysis explored temperature changes in the three days before admission for neutropenic sepsis. 1,755 temperature readings were returned by healthy participants and 1,765 by chemotherapy patients. Mean participant temperature was $36.16 \mathrm{C}$ (95\% CI 36.07-36.26) in healthy participants and 36.32C (95\% CI 36.18-36.46) in chemotherapy patients. Healthy participant within-person variability was $0.40 \mathrm{C}(95 \% \mathrm{CI} 0.36-0.44)$ and between-person variability was $0.26 \mathrm{C}(95 \% \mathrm{CI}$ 0.16-0.35). Chemotherapy patient within-person variability was $0.39 \mathrm{C}(95 \% \mathrm{CI} 0.34-0.44)$ and between-person variability was $0.34 \mathrm{C}(95 \% \mathrm{CI} 0.26-0.48)$. Thus, use of a population mean rather than personalised baselines is probably sufficient for most clinical purposes as between-person variability is not large compared to within-person variability. Standardised guidance and provision of thermometers to patients might help to improve recording and guide management.

Keywords: temperature variability, chemotherapy, neutropenic sepsis, thermometry.

\section{Introduction}




\section{Variability in biological measurements}

Variability in biological measurements can be conceptualised as arising from two distinct sources of variation: within-person variability, and between-person variability[2]. Withinperson variability describes how stable a measure is when recorded multiple times on a single subject, whereas between-person variability describes how measurements from different subjects vary from each other. For example, adult standing height has very low within-person variability (it changes only slightly when measured multiple times on the same person), but has considerable between-person variability (different people in a population will have a variety of heights). If we are considering whether to use a population-based or a personalised cut-off for temperature, it is important to consider these potential sources of variability. If within-person variability is low compared to between-person variability, then personalised cut-offs may be worth exploring, as each individual is likely to have a reliable "set point". However, if within- 
person variability is similar to, or higher than, between-person variability, then the benefit of personalised cut-offs is much reduced.

\section{Existing research on temperature and variability}

Early work on temperature monitoring by Wunderlich[3] defined the physiological normal point of temperature at $37^{\circ} \mathrm{C}$. This study considered temperatures within a range $36.2-37.5^{\circ} \mathrm{C}$ as normal, with significant within-person variation, up to $0.5^{\circ} \mathrm{C}$, observed. Temperatures of $38^{\circ} \mathrm{C}$ or higher were considered 'probably febrile'. More recent research found the mean temperature to be $36.8^{\circ} \mathrm{C}$, with the upper limit of normal considered as $37.7^{\circ} \mathrm{C}$, though they observed significant diurnal variation[4]. A large systematic review of 27 studies published between 1935 and 1998 found that the mean value for oral temperature was $36.4^{\circ} \mathrm{C}$ and that temperature is dependent on the site at which it is measured, although the review did not assess variability[5]. There are other papers in which between-person variability in temperature is reported. However, aside from concluding that there was no 'normal' baseline temperature in their population[6], there is no additional discussion of baseline temperature variation (which could be used to determine individual definitions of 'fever') in the current literature.

\section{Neutropenic sepsis in chemotherapy}

Neutropenic sepsis is a potentially fatal complication of chemotherapy treatment, with mortality rates between 2 and 21\%[7]. Rapid detection is important so that immediate antibiotic treatment can be commenced[8]. Current NICE guidelines define neutropenic sepsis as an absolute neutrophil count of $\leq 0.5 \times 10^{9} / \mathrm{L}$ with symptoms, which may include a fever over $38^{\circ} \mathrm{C}$ or signs of sepsis[7]. Neutropenia is a common consequence of chemotherapy treatment, but 
does not itself cause any symptoms. Usual clinical features of sepsis may not be present in patients in whom the systemic inflammatory response to infection is attenuated[9], and so temperature is a key diagnostic indicator for this condition. However, there is no consensus definition of fever, and diagnostic protocols vary between centres: a survey of 55 centres found more than 10 different definitions of fever, with temperature cut-offs ranging from 37.0 to $39.0^{\circ} \mathrm{C}[10]$. Patients receiving chemotherapy may be asked to self-monitor their temperature at home to increase the likelihood of early detection and treatment[11]. However, guidance on how often and when to monitor temperature varies between trusts and specialities, and evidence to guide frequency of self-monitoring is lacking[10]. There is little research on normal variation of body temperature during chemotherapy treatment, and whether a universal temperature cutoff to identify fever is valid or whether establishing personalised temperature baselines would be more appropriate.

Given the scarcity of data on temperature variability in both healthy and disease states, this study aimed to determine between-person and within-person variability of temperature in healthy adults and in patients receiving chemotherapy. The work in chemotherapy patients was also designed to assess the feasibility of a larger study to ascertain the most useful temperature cut-offs for predicting neutropenic sepsis, in order to inform guidelines and practice for patients and clinicians.

\section{Methods}

Patient and public involvement 
One of the authors (HFA) attended a local breast cancer peer support group of 14 women who had completed or were undergoing breast cancer treatment, the majority of whom had received chemotherapy, in order to investigate their experiences of temperature monitoring and their opinions on the planned research at the protocol design stage (October 2014). There was significant variation of temperature monitoring frequency, ranging from only if they felt unwell, every few days or once per day, to one person who recorded three times a day to establish a baseline and any deviation from it (acknowledging this was her personal choice rather than specific advice from the medical team involved in her care). Some, however, did not realise that temperature monitoring was important or necessary. No patient had been provided with a thermometer, but were instead advised either to buy one or use the one they had already, and no-one recalled receiving any guidance on the type of thermometer to use. One person had used a mercury thermometer she had owned for years. Group members were in strong agreement with the need for further research in this area, indicating that participation would not be over-burdensome, and they helped develop details of the planned research (for example, recommending a printed booklet to record temperature rather than online/text message monitoring).

\section{Measurements in healthy adults}

We invited healthy adults to take part in the study between August and November 2015, using advertisements placed around university premises. Participants had to be aged 16 years or over, not taking any medication regularly that could potentially affect their body temperature (for example paracetamol or non-steroidal anti-inflammatory drugs (NSAIDs)), and not be taking part in any interventional study. All participants gave written informed consent. 
We collected basic details from each participant, including age, sex, medical conditions, and any regular medication. We provided each participant with a digital electronic thermometer (Omron Eco Temp Smart, OMRON Healthcare UK Ltd., Milton Keynes, UK), and a paper diary in which to record their temperature readings. We asked participants to use the thermometer to measure their oral temperature up to three times a day for three weeks. The diary included space for the date and time of the recording, the measured temperature, and any additional information (e.g. regarding concurrent symptoms such as feeling feverish) that the participant felt was important. We also asked participants to record any use of antipyretic medication (paracetamol or NSAIDs), although we did not ask them to record whether this was taken for fever or pain relief. Participants returned completed diaries to the study team in prepaid envelopes.

In addition to the manufacturer's instruction leaflet, we provided additional guidance to participants at the start of the study and in their diaries, which covered correct use of the thermometer, including guidance not to take measurements within 30 minutes of exercise, bathing, or drinking hot or cold beverages. We also provided guidance on the normal range of temperature and encouraged participants to seek medical advice in the usual way if they were concerned about abnormal temperature readings or felt unwell; however, we also had a medical practitioner on the study team who was available by phone or email to answer queries from study participants. Participants received up to three reminders via phone or e-mail if they hadn't returned their diaries. 


\section{Measurements in patients receiving chemotherapy}

Patients were recruited from the Day Treatment Unit, Churchill Hospital, Oxford, between November 2015 and June 2016. The Day Treatment Unit is an outpatient unit where patients undergoing treatment for oncological or haematological cancers receive chemotherapy. Patients were eligible if they were aged 16 or over, undergoing any chemotherapy treatment (all of which is known to pose some risk for neutropenic sepsis), had access to a thermometer, and were planning to self-monitor temperature during their chemotherapy cycle (a 'cycle' refers to the time period between chemotherapy infusions, usually three weeks, during which they might be at risk of neutropenic sepsis). Potential participants were first approached by a member of the clinical team to ask their permission to be approached by the research team, and were then given a verbal explanation about the study along with written information. We recorded the number of patients approached, and their reasons for declining participation if applicable. All participants gave written informed consent.

We collected details from each participant and also sought permission to view their medical records to corroborate information. We recorded demographic details including age and sex, and recorded the current cancer diagnosis, chemotherapy regimen, cycle duration, past medical history, and current medications. Patients were provided with a variable number of diaries based on their expected future number of cycles (one diary per cycle). The diaries were similar to those used by the healthy volunteers, with additional space to provide details of any hospital admissions during the recording period, as well as a separate section for recording details of the thermometer(s) used to measure temperature (including brand, model, location of purchase, duration of ownership, and site of temperature measurement). As guidance given to patients on temperature monitoring during chemotherapy treatment varies, we asked them not to change 


\section{Data analysis}

their existing or planned practice but simply to record their temperature in the diary when they were checking it routinely. Participants were explicitly told not to contact the research team in the event of being unwell but to seek medical advice as instructed by their supervising clinicians. Participants returned completed diaries (anonymised using participant-specific codes) to the study team in prepaid envelopes. Patients were contacted up to two times if no diaries were received by the study team. We checked medical records retrospectively for the period of temperature recording and any intervening periods to ensure there had been no admissions for neutropenic sepsis not recorded by the participants themselves. In addition to what was recorded in the diaries, we retrospectively reviewed the electronic medical records for all those who returned diaries. We counted an admission as due to neutropenic sepsis when this was recorded as such in the patient booklet, or when 'neutropenic sepsis', 'febrile neutropenia', or similar were included as part of the diagnosis in the discharge letter or other patient notes.

For the primary analysis, we included all temperature measurements where there were no obvious protocol deviations recorded in the temperature diaries. For healthy patients, protocol deviations were identified when the diary indicated that measurements were made after bathing, exercise, or consumption of hot or cold drinks, as the thermometer instructions provided in the diary specifically advised against these. The diary for chemotherapy patients did not include these instructions, so they were not applied to the chemotherapy results. Primary outcome was within-person and between-person variability in both healthy and chemotherapy participant groups, as measured by temperature means and within-person and 
between-person standard deviation. Student's t-test was used for comparisons between groups, and we used the widely accepted value of $\mathrm{p}<0.05$ as our indicator of statistical significance.

We planned pre-specified secondary analysis in the chemotherapy patient group to explore descriptively and graphically how patient and disease characteristics, including age, sex, underlying medical conditions (using the Charlson Comorbidity Index)[12], cancer type (especially haematological vs. oncological due to the differing incidence of neutropenic sepsis in these groups), and drug treatment might affect temperature variability. Particularly, we wanted to investigate the effect on temperature of anti-pyretic medication (including paracetamol and NSAIDs), anti-infective medication (used occasionally during certain chemotherapeutic regimens), and the use of Granulocyte Colony Stimulating Factor (G-CSF) (which aims to reduce the incidence of neutropenic sepsis)[13]. It is known that body temperature variation can be impaired at extremes of age[14], and so we performed a sensitivity analysis to test whether exclusion of any very elderly patients influenced the overall results. For healthy participants, we also performed a sensitivity analysis excluding patients with underlying medical conditions which might plausibly affect their temperature. Sensitivity analysis was performed using Student's t-test.

In order to investigate the changes in temperature around the period of an episode of neutropenic sepsis, we conducted descriptive and graphical analyses of the temperature readings three days either side of a hospital admission for neutropenic sepsis, and sensitivity analysis of overall results excluding these readings. Information provided by the participants on thermometer type was used to compare temperature differences between place of purchase of thermometer or site of temperature measurement. 
Statistical analysis was overseen by a senior medical statistician (RJS).

\section{Sample size}

Based on the formulae of Ahn and Fessler[15], we calculated that we would need 40 patients with 40 or more readings each to allow estimation of standard deviation with precision approximately $+/-11 \%$. To allow for missing data and for loss to follow-up, we therefore sought to recruit 50 participants in each of the healthy and chemotherapy groups. Unfortunately due to staffing constraints we were unable to meet our pre-planned recruitment target; however this was made up for by a large number of readings from some participants.

\section{Ethical approval}

The study carried out in healthy adults received a favourable ethical opinion from the University of Oxford Central University Research Ethics Committee (approval number MSIDREC-C1-2015-144). The study carried out in chemotherapy patients received a favourable ethical opinion from North East - York Research Ethics Committee (approval number 15/NE/0267).

\section{Results}

\section{Included participants}

We recruited 44 healthy volunteers, 29 of whom returned diaries. 67 patients receiving chemotherapy were eligible to participate during the times the research team were present in the Day Treatment Unit, of whom 43 were recruited to the study. Of the remaining 24, eight 
declined to discuss with the research team following approach by the clinical team, one was excluded by the clinical team as unsuitable to speak to the research team, four patients agreed to speak with the research team but left the unit before this could occur, and 11 declined to participate following provision of study information. Of the 43 participants recruited, 23 (7 haematology and 16 oncology) returned diaries for a total of 65 chemotherapy cycles. The average number of diaries returned per patient was 2.8 , with a range of 1-6.

Attempts were made to contact those who did not return booklets. One patient stated the booklet was lost in postage, three patients stated they had lost booklets or filling them in was too much effort, and we were unable to contact the remaining patients despite two attempts. Of the patients we failed to contact, investigation of their medical notes revealed that six changed chemotherapy regimens following recruitment, either due to side-effects or poor response, one underwent assessment for a clinical trial, one changed treatment following autologous stem cell transplantation, and one underwent surgery shortly after recruitment, subsequently developing complications resulting in several intensive care admissions and a prolonged stay in hospital. A further uncontactable patient died within two months of recruitment.

Table 1 summarises demographic and medical information of those who did and did not return diaries. Of healthy volunteers who returned diaries, the median age was 27 years (range 19-60 years), with 14 males and 14 females (one sex not reported). Of chemotherapy patients who returned diaries, the median age was 63 years (range 42-90 years), with 13 males and 10 females.[Table 1 near here] 


\section{Within-person and between-person variability in temperature}

A total of 1,755 temperature readings were returned from the healthy participants and 1,765 from the chemotherapy patients. Protocol deviations were identified for 17 temperature readings from healthy patients, leaving 1738 readings in the primary analysis. Readings were excluded for report of recent cold (6 readings) or hot beverage consumption ( 2 readings), recent exercise (6 readings), and recent bathing (3 readings). Haematology patients recorded for more days and provided more readings per day than oncology patients (Table 2). The mean of participants' mean temperatures was higher in patients receiving chemotherapy than in healthy participants $\left(36.32^{\circ} \mathrm{C}(95 \%\right.$ CI $36.18-36.46)$ compared to $36.16^{\circ} \mathrm{C}(95 \%$ CI $\left.36.07-36.26)\right)$ but this was just outside of statistical significance $(\mathrm{p}=0.06)$ (see Figure 1). Mean within-person variability was higher than between-person variability in both healthy and chemotherapy participants (Table 2), although for the latter group the difference was not large compared to the width of confidence intervals. The range of temperature readings for all participants included the overall sample mean temperature, with the exception of one patient who only provided 11 temperature readings which ranged from $36.5-37.1^{\circ} \mathrm{C}$ (see Figure 2). [Table 2 near here]

\section{How participant characteristics explain variability in patients receiving chemotherapy}

We conducted planned sensitivity and subgroup analyses by age, sex, cancer type, cancer treatment, comorbidity, and concomitant medication use in the chemotherapy patient group.

Mean temperature was higher with increasing age but this was not statistically significant (correlation coefficient $=0.30, p=0.17$ ). In a sensitivity analysis excluding the 3 most elderly 
patients from the analysis (aged 83, 83 and 90), mean chemotherapy patient temperature was $36.28^{\circ} \mathrm{C}\left(95 \% \mathrm{CI} 36.13-36.43^{\circ} \mathrm{C}, \mathrm{n}=20\right)$.

Men and women had similar mean temperature $\left(36.27^{\circ} \mathrm{C}(95 \% \mathrm{CI} 36.05-36.49)\right.$ vs. $36.38^{\circ} \mathrm{C}$ (95\%CI 36.24-36.52), $\mathrm{p}=0.45)$, similar within-person variability $\left(0.39^{\circ} \mathrm{C}(95 \% \mathrm{CI} 0.33-0.45)\right.$ vs. $\left.0.40^{\circ} \mathrm{C}(95 \% \mathrm{CI} 0.32-0.48)\right)$ and between-person variability $\left(0.40^{\circ} \mathrm{C}(95 \% \mathrm{CI} 0.29-0.67)\right.$ vs. $\left.0.23^{\circ} \mathrm{C}(95 \% \mathrm{CI} 0.16-0.41)\right)$

Mean temperature was higher in haematology patients than oncology patients but this was not statistically significant $\left(36.49^{\circ} \mathrm{C}(95 \%\right.$ CI $36.34-36.63, \mathrm{n}=7)$ vs. $36.25^{\circ} \mathrm{C}(95 \%$ CI $36.07-36.42$, $\mathrm{n}=16$ ), $\mathrm{p}=0.11$ ). There were too few patients per cancer subtype to determine whether temperature varied between subtypes (Appendix Table 1).

\begin{abstract}
Although we had planned to investigate whether use of G-CSF had any effect on temperature, this was not part of the treatment regime for any of the participants who returned diaries.

We found no consistent correlation between day of the chemotherapy cycle and temperature: mean correlation coefficient for 64 cycles $=0.06$, standard deviation $=0.40$ (one cycle had only two temperature readings, so correlation coefficient was not calculated for this cycle).
\end{abstract}

Mean temperature increased with Charlson Comorbidity Index and Charlson Age-Comorbidity Index, but this was not statistically significant (Charlson Comorbidity Index, correlation coefficient $=0.26, p=0.23$; Charlson Age-Comorbidity Index, correlation coefficient $=0.36$, $\mathrm{p}=0.09$ ). 
Of all the individual temperature readings collected, 338/1765 (19.2\%) were recorded with the anti-inflammatory/paracetamol box checked in the temperature diary. The mean of these temperature readings was $36.63^{\circ} \mathrm{C}\left(95 \% \mathrm{CI} 35.59-36.67^{\circ} \mathrm{C}, \mathrm{n}=338\right)$, compared to $36.35^{\circ} \mathrm{C}(95 \%$ CI $36.33-36.38^{\circ} \mathrm{C}, \mathrm{n}=1427$ ) without $(\mathrm{p}<0.001)$. A sensitivity analysis excluding these readings reduced the mean of individual temperature readings to $36.35^{\circ} \mathrm{C}(95 \%$ CI $36.33-36.38)$, compared with the whole dataset mean of $36.41^{\circ} \mathrm{C}\left(95 \%\right.$ CI $\left.36.39-36.43^{\circ} \mathrm{C}, \mathrm{p}=0.002\right)$. Four haematology patients were treated with medication to prevent viral or fungal infection (aciclovir and fluconazole respectively). These patients had a slightly higher mean temperature $\left(36.57^{\circ} \mathrm{C}\left(95 \% \mathrm{CI} 36.36-36.78^{\circ} \mathrm{C}\right)\right.$ vs. $36.27^{\circ} \mathrm{C}\left(95 \% \mathrm{CI} 36.12-36.42^{\circ} \mathrm{C}\right)$, although this was nonsignificant.

\section{Changes in temperature before unplanned admission for sepsis}

Five of 23 patients (21.7\%) experienced an episode of neutropenic sepsis during their treatment (three oncology, two haematology), two of whom had episodes during the period they were recording their temperature (these two patients, one haematology and one oncology, experienced two unplanned admissions for neutropenic sepsis each during data collection). Both patients were able to record temperatures in the days leading up to admission and during the episode of sepsis on all four occasions (see Figure 3). In these patients, temperature was higher in the three days prior to admission for neutropenic sepsis compared to their baseline (excluding episode of neutropenic sepsis) $\left(36.23^{\circ} \mathrm{C}\right.$ vs. $36.05^{\circ} \mathrm{C}(\mathrm{p}=0.33)$ and $36.94^{\circ} \mathrm{C}$ vs. $\left.36.70^{\circ} \mathrm{C}(\mathrm{p}=0.10)\right)$, but with very small numbers of readings for this period in both patients ( $n=6$ and $n=8$ respectively). In one patient, temperature during periods of neutropenic sepsis never rose higher than $37.5^{\circ} \mathrm{C}$. A sensitivity analysis excluding temperature values within three days either side of a hospital admission for neutropenic sepsis had minimal effect on overall 
mean temperature for chemotherapy patients (mean temperature $36.31^{\circ} \mathrm{C}(95 \%$ CI 36.17 $\left.36.45^{\circ} \mathrm{C}\right)$ ).

\section{Current patterns of thermometer use amongst chemotherapy patients}

Twelve patients (55\%) used Omron Digital thermometers provided by the hospital, whilst ten (45\%) had purchased thermometers from high street stores. One participant did not provide any information regarding the thermometer used. Mean temperature was similar between patients using purchased or hospital-provided thermometers $\left(36.37^{\circ} \mathrm{C}\left(95 \%\right.\right.$ CI $36.21-36.52^{\circ} \mathrm{C}$, $\mathrm{n}=11)$ vs. $36.27^{\circ} \mathrm{C}\left(95 \%\right.$ CI $\left.\left.36.06-36.52^{\circ} \mathrm{C}, \mathrm{n}=11\right)\right)$, and in subgroup analysis by location of testing (axillary $36.30^{\circ} \mathrm{C}(95 \%$ CI $36.20-36.40, \mathrm{n}=2)$; aural $36.49^{\circ} \mathrm{C}\left(95 \%\right.$ CI $36.16-36.82^{\circ} \mathrm{C}$, $\mathrm{n}=4)$; oral $\left.36.28^{\circ} \mathrm{C}\left(95 \% \mathrm{CI} 36.09-36.46^{\circ} \mathrm{C}, \mathrm{n}=15\right)\right)$. The number of patients in each group was not deemed sufficiently large to perform a Student's t-test.

\section{Discussion}

\section{Statement of principal findings}

Body temperature varied between $34.3^{\circ} \mathrm{C}$ and $38.7^{\circ} \mathrm{C}$ in healthy participants, and in chemotherapy patients without neutropenic sepsis. However, in both groups this variation was as much due to variation within individuals (over time) as to variation between individuals, and the range of temperature readings for all but one participant included the sample mean temperature. There is, therefore, no strong case, within the limitations of this dataset, for individualising thresholds for monitoring. During four episodes of neutropenic sepsis in two patients, temperature was higher than the patient's baseline in the period preceding the 
diagnosis. Frequency of temperature monitoring during chemotherapy ranged from 0 to 6 times per day, and there was also variation in the type of thermometer used to provide measurements, and whether this was provided by the hospital.

\section{Strengths and weaknesses of the study}

This is the first study to assess variation of temperature during chemotherapy treatment, with a parallel study in healthy adults using similar methods. We deliberately did not impose a large number of constraints in terms of type or age of thermometer to use, frequency of testing, or time of day. The advantage of this approach is to obtain real-life results that reflect clinical practice, however the corresponding limitation is that we cannot quantify in the same study how far variability could be reduced by enforcing a more uniform practice: for example, giving strict guidance to chemotherapy patients on timing of measurement in relation to hot drinks or exercise, or on time of day for measurement to take place. Data were collected at a different time of year for healthy participants (August to November) compared to chemotherapy patients (November to June) and season may also affect temperature measurements, although the reported direction of change is conflicting between studies[16]. We had a relatively modest return rate of diaries from participants recruited $(66 \%$ for healthy and $53 \%$ for chemotherapy participants) which may reflect this being a group of patients who have multiple other concerns whilst undergoing chemotherapy treatment; it is possible that the group who returned diaries may be in better health and therefore not typical of the whole population receiving chemotherapy, or episodes of neutropenic sepsis may have been more frequent in diary nonreturners. It is possible that patients may have altered the frequency of monitoring temperature due to taking part in the study, for example recording more frequently. Analyses reported for pre-specified secondary analyses of chemotherapy participant data should be interpreted with 
caution due to small numbers in each group. Our healthy participants, recruited in a university setting, were in general younger than the chemotherapy patients, so that comparisons between the groups should be made with caution. However, our main findings are comparisons made within groups (within healthy participants, and within chemotherapy patients) and therefore unconfounded by age.

\section{Strengths and weaknesses in relation to other studies}

Mean temperatures of $36.16^{\circ} \mathrm{C}$ and $36.32^{\circ} \mathrm{C}$ in our two groups were both slightly lower than that found in the systematic review of temperature (mean $36.4^{\circ} \mathrm{C}$ )[5]. The systematic review included studies published as early as 1935, and earlier studies tend to report higher mean temperatures; it is not known whether this is due to changes in the population over time, or changes in thermometer technology. We selected the thermometer we gave to healthy participants as a widely available model suitable for oral use, and it is possible that a fixed measurement bias might account for the lower temperature in healthy participants.

Two studies which monitored temperature in patients with cancer (11 patients with advanced cancer[17] and nine in breast cancer survivors experiencing hot flushes)[18] found wide within-person variability; we found within-person variability to be similar between chemotherapy and healthy participants. A previous study of 320 participants found that the febrile response to infection can be reduced with age[14]; we found no significant correlation with increasing age in our study. The number of patients in our study experiencing episodes of neutropenic sepsis $(21.7 \%)$ was at the upper end of that reported in the literature for solid tumours (13-21\%)[19], perhaps because our study population, by design, included both 
oncology and haematology patients, the latter group of whom might be expected to have more episodes of neutropenic sepsis.

\section{Possible explanations and implications for clinicians and policymakers}

Setting a treatment threshold for detection of neutropenic sepsis is difficult because temperatures vary even in patients without sepsis. Our results indicate that this cannot be solved by using different thresholds for different patients, because the variation is as much, or more, within individuals than between individuals. We find that the low thresholds used by some UK centres would be exceeded occasionally even by healthy participants, who recorded many temperatures greater than $37.0^{\circ} \mathrm{C}$ (the lowest threshold in use in a UK survey)[10] and a few readings greater than $38.0^{\circ} \mathrm{C}$. On the other hand, the highest thresholds in use in the survey, $39.0^{\circ} \mathrm{C}$, would not have been adequate to detect the cases of neutropenic sepsis in our study as no patient or healthy volunteer recorded a temperature above this at any point. Threshold setting, therefore, remains challenging. While it would be inappropriate to draw firm conclusions from the very small number of episodes of neutropenic sepsis recorded in our study, these patients did have a raised mean temperature in the three days prior to admission (and therefore presumed diagnosis) compared to their baseline. This, in retrospect, raises the possibility of a role for establishing a personalised baseline using rolling averages. Elevated temperature is already the most common trigger for seeking medical help in asymptomatic patients[11], so consistent measurement might flag a problem sooner than with current practice, though we should emphasise that there should not be over-reliance on temperature alone as a sign of neutropenic sepsis[9]. The variation we observed in chemotherapy patients in device, testing frequency, and measurement site (axillary testing is known to give lower temperatures)[5] leaves open the possibility that variability could be reduced by 
standardisation: for example, providing all patients with an identical thermometer of known reliability, and providing guidance on testing frequency. It is also important to acknowledge that there may be differences in suitable recommendations between different cancer types. At present, clinicians should consider asking patients the site of measurement when assessing the significance of a high or normal temperature reading.

\section{Unanswered questions and future research}

The consistency and precision of current thermometers, and the potential benefit of standardising on a single, well-validated thermometer, therefore requires further research. There is no current consensus about the temperature threshold to use when monitoring patients for potential neutropenic sepsis; data such as ours needs to be supplemented with more data about patients with neutropenic sepsis, and in particular temperature changes in the hours prior to admission for sepsis, before an evidence-based threshold can be determined. Several additional patients did have episodes of neutropenic sepsis outside of our data collection period, and so recruiting patients at commencement of chemotherapy and recording temperature for the duration of treatment would yield more power for future analyses, and this would be a feasible study. The wide range of thresholds in use even within a single health service (UK NHS) suggests an evidence gap that urgently needs addressing.

\section{Acknowledgements}

We would like to thank David Mant who conceived the idea for the study in chemotherapy patients; Louis Werner for healthy participant data collection; Evie Monaghan for data entry; and the Bosom Friends breast cancer support group for sharing their experiences and their contributions to study design. 


\section{Funding}

This research received no specific grant from any funding agency in the public, commercial or not-for-profit sectors. A small internal fund was attached to a medical student placement, which provided funds for thermometers for the healthy participant group.

\section{Competing interests}

None declared.

\section{Contributorship statement}

DM, HFA, SF, and RJS conceived the idea for the study, and designed the study with contributions from EF, VW and TL. LW, HFA, JSF, and GB acquired the data. SF, RJS, JSF, GB and HFA analysed the data and drafted the manuscript, with critical revisions from EF, VW and TL. All authors approved the final version to be published.

\section{Data sharing statement}

Full anonymised dataset available on request from the corresponding author. 


\section{References}

1 Kelly G. Body temperature variability (Part 1): a review of the history of body temperature and its variability due to site selection, biological rhythms, fitness, and aging. Altern Med Rev 2006; 11(4): 278-93.

2 Stevens RJ, Oke J, Perera R. Statistical models for the control phase of clinical monitoring. Stat Methods Med Res 2010;19(4):394-414.

3 Wunderlich CA, Seguin E. Medical thermometry, and human temperature. New York, U.S.A.: William Wood \& Company 1871.

4 Mackowiak PA, Wasserman SS, Levine MM. A critical appraisal of 98.6 degrees F, the upper limit of the normal body temperature, and other legacies of Carl Reinhold August Wunderlich. JAMA 1992;268(12):1578-80.

5 Sund-Levander M, Forsberg C, Wahren LK. Normal oral, rectal, tympanic and axillary body temperature in adult men and women: a systematic literature review. Scand J Caring Sci 2002;16(2):122-8.

6 Horvath SM, Menduke H, Piersol GM. Oral and rectal temperatures of man. Journal of the American Medical Association 1950;144(18):1562-5.

7 Neutropenic Sepsis: Prevention and Management of Neutropenic Sepsis in Cancer Patients. National Institute for Health and Care Excellence: Guidance 2012.

8 National Confidential Enquiry into Patient Outcome and Death. For better, for worse? A review of the care of patients who died within 30 days of receiving systemic anti-cancer therapy. London, UK 2008.

9 Clarke RT, Jenyon T, van Hamel Parsons V, et al. Neutropenic sepsis: management and complications. Clin Med (Lond) 2013;13(2):185-7.

10 Clarke RT, Warnick J, Stretton K, et al. Improving the immediate management of neutropenic sepsis in the UK: lessons from a national audit. Br J Haematol 2011;153(6):773-9.

11 Clarke RT, Bird S, Kakuchi I, et al. The signs, symptoms and help-seeking experiences of neutropenic sepsis patients before they reach hospital: a qualitative study. Support Care Cancer 2015;23(9):2687-94.

12 Charlson ME, Pompei P, Ales KL, et al. A new method of classifying prognostic comorbidity in longitudinal studies: development and validation. J Chronic Dis 1987;40(5):373-83.

13 Aapro MS, Bohlius J, Cameron DA et al. 2010 update of EORTC guidelines for the use of granulocytecolony stimulating factor to reduce the incidence of chemotherapy-induced febrile neutropenia in adult patients with lymphoproliferative disorders and solid tumours. European Journal of Cancer 2011;47(1):832. 
14 Roghmann MC, Warner J, Mackowiak PA. The relationship between age and fever magnitude. Am J Med Sci 2001;322(2):68-70.

15 Ahn S, Fessler JA. Standard errors of mean, variance, and standard deviation estimators. University of Michagan report 2003.

16 Kelly GS. Body temperature variability (Part 2): masking influences of body temperature variability and a review of body temperature variability in disease. Altern Med Rev 2007;12(1):49-62.

17 Bailleul F, Levi F, Reinberg A, et al. Interindividual differences in the circadian hematologic time structure of cancer patients. Chronobiol Int 1986;3(1):47-54.

18 Carpenter JS, Gilchrist JM, Chen K, et al. Hot flashes, core body temperature, and metabolic parameters in breast cancer survivors. Menopause 2004;11(4):375-81.

19 Weycker D, Li X, Edelsberg J, et al. Risk and Consequences of Chemotherapy-Induced Febrile Neutropenia in Patients With Metastatic Solid Tumors. J Oncol Pract 2015;11(1):47-54. 
Figure 1 Distribution of mean temperature in healthy and chemotherapy participants.

Figure 2 Temperatures recorded by healthy participants (upper graph), chemotherapy patients (lower graph, haematology (light grey) and oncology (dark grey) patients), ordered by participant mean. For each participant, centre line denotes the median, boxes denote interquartile range; whiskers denote $1.5 \mathrm{x}$ the interquartile range above or below the upper and lower quartile respectively; dots are outliers outside of the whisker definition. Solid vertical line denotes the sample mean, with dotted vertical lines representing $95 \%$ confidence intervals.

Figure 3 Individual temperature monitoring graphs for chemotherapy patients who experienced neutropenic sepsis. Solid box indicates the period of the admission for neutropenic sepsis and dashed box three days either side. 
Table 1 Demographic information of participants who did and did not return diaries.

\begin{tabular}{|c|c|c|c|c|}
\hline & \multicolumn{2}{|c|}{ Healthy participants } & \multicolumn{2}{|c|}{ Chemotherapy patients } \\
\hline & $\begin{array}{l}\text { Included (\%) } \\
\mathrm{N}=29\end{array}$ & $\begin{array}{l}\text { Non-returners } \\
(\%) \\
\mathrm{N}=13\end{array}$ & $\begin{array}{l}\text { Included }(\%) \\
\mathrm{N}=23\end{array}$ & $\begin{array}{l}\text { Non-returners } \\
(\%) \\
\mathrm{N}=20\end{array}$ \\
\hline Male & $14(48.3)$ & $4(30.8)$ & $13(56.5)$ & $9(45.0)$ \\
\hline Female & $14(48.3)$ & $8(61.5)$ & $10(43.5)$ & $11(55.0)$ \\
\hline Not reported & $1(3.4)$ & $1(7.7)$ & $0(0.0)$ & $0(0.0)$ \\
\hline $16-29$ & $16(55.2)$ & $9(69.2)$ & $0(0.0)$ & $2(10.0)$ \\
\hline $30-49$ & $11(37.9)$ & $1(7.7)$ & $5(21.7)$ & $3(15.0)$ \\
\hline $50-69$ & $1(3.4)$ & $0(0.0)$ & $12(52.2)$ & $11(55.0)$ \\
\hline$\geq 70$ years & $0(0.0)$ & $0(0.0)$ & $6(26.1)$ & $4(20.0)$ \\
\hline Not reported & $1(3.4)$ & $3(23.1)$ & $0(0.0)$ & $0(0.0)$ \\
\hline Haematology & $\mathrm{N} / \mathrm{a}$ & $\mathrm{N} / \mathrm{a}$ & $7(30.4)$ & $5(25.0)$ \\
\hline Oncology & $\mathrm{N} / \mathrm{a}$ & $\mathrm{N} / \mathrm{a}$ & $16(69.6)$ & $15(75.0)$ \\
\hline $\begin{array}{l}\text { Pre-existing } \\
\text { comorbidities* }\end{array}$ & $0(0.0)$ & $0(0.0)$ & $9(39.1)$ & $5(25.0)$ \\
\hline $\begin{array}{l}\text { Taking any } \\
\text { regular } \\
\text { prescribed } \\
\text { medication }\end{array}$ & $4(13.8)$ & $1(6.6)$ & $13(56.5)$ & $13(65.0)$ \\
\hline
\end{tabular}

*as defined by Charlson Comorbidity Index[12] 
Table 2 Temperature recordings for healthy participants and patients receiving chemotherapy.

\begin{tabular}{|c|c|c|c|c|c|}
\hline & & $\begin{array}{l}\text { Healthy } \\
(n=29)\end{array}$ & $\begin{array}{l}\text { All chemotherapy } \\
\text { patients } \\
(\mathrm{n}=23)\end{array}$ & $\begin{array}{l}\text { Haematology } \\
(n=7)\end{array}$ & $\begin{array}{l}\text { Oncology } \\
(n=16)\end{array}$ \\
\hline \multirow{5}{*}{ Recordings } & Total number & 1755 & 1765 & 1100 & 665 \\
\hline & $\begin{array}{l}\text { Median days } \\
\text { recorded } \\
\text { (mean) }\end{array}$ & $21(20.3)$ & $51(56.6)$ & $115(100.6)$ & $27.5(37.3)$ \\
\hline & $\begin{array}{l}\text { Median number } \\
\text { of readings } \\
\text { (mean) }\end{array}$ & $63(60.5)$ & $51(76.7)$ & $138(157.1)$ & $40(41.6)$ \\
\hline & $\begin{array}{l}\text { Median number } \\
\text { of readings/day } \\
\text { (mean) }\end{array}$ & $3(2.98)$ & $1(1.3)$ & $1.4(1.5)$ & $1(1.3)$ \\
\hline & $\begin{array}{l}\text { Range of } \\
\text { readings/day }\end{array}$ & $0-4$ & $0-6$ & $0-4$ & $0-6$ \\
\hline \multirow{4}{*}{$\begin{array}{l}\text { Temperature } \\
\text { (degrees } \\
\text { Celsius) }\end{array}$} & $\begin{array}{l}\text { Mean per } \\
\text { person }(95 \% \\
\text { CI) }\end{array}$ & $\begin{array}{l}36.16 \\
(36.07- \\
36.26) \\
\end{array}$ & $\begin{array}{l}36.32(36.18- \\
36.46)\end{array}$ & $\begin{array}{l}36.49 \\
(36.34-36.63)\end{array}$ & $\begin{array}{l}36.25(36.07- \\
36.42)\end{array}$ \\
\hline & $\begin{array}{l}\text { Within-person } \\
\text { variability }(95 \% \\
\text { CI) }\end{array}$ & $\begin{array}{l}0.40(0.36- \\
0.44)\end{array}$ & $0.39(0.34-0.44)$ & $0.41(0.30-0.52)$ & $\begin{array}{l}0.39(0.33- \\
0.44)\end{array}$ \\
\hline & $\begin{array}{l}\text { Between-person } \\
\text { variability (95\% } \\
\text { CI) }\end{array}$ & $\begin{array}{l}0.26(0.16- \\
0.35)\end{array}$ & $0.34(0.26-0.48)$ & $0.19(0.13-0.43)$ & $\begin{array}{l}0.36(0.27- \\
0.56)\end{array}$ \\
\hline & Range & $34.1-38.4$ & $34.3-38.7$ & $34.3-38.7$ & $34.4-38.1$ \\
\hline
\end{tabular}


Appendix Table 1 Patients receiving chemotherapy divided by cancer type

\begin{tabular}{|l|l|}
\hline Cancer type & $\mathrm{n}$ \\
\hline Haematological & 7 \\
Lymphoma & 2 \\
Leukaemia & 5 \\
\hline Oncological & 16 \\
Oesophageal & 3 \\
Breast & 2 \\
Ovarian & 1 \\
Testicular & 2 \\
Colorectal & 5 \\
Prostate & 1 \\
Fibrosarcoma & 1 \\
Chordoma & 1 \\
\hline
\end{tabular}




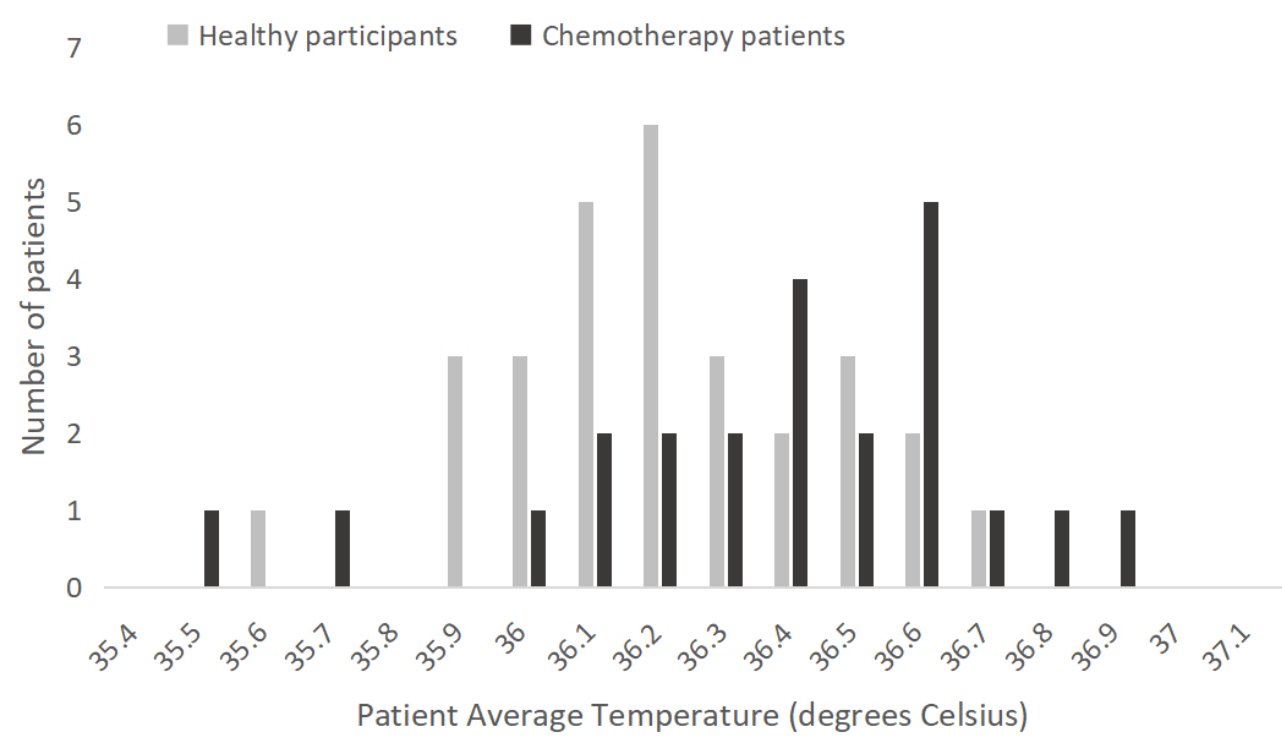

Figure 1 - Distribution of mean temperature in healthy and chemotherapy participants. 

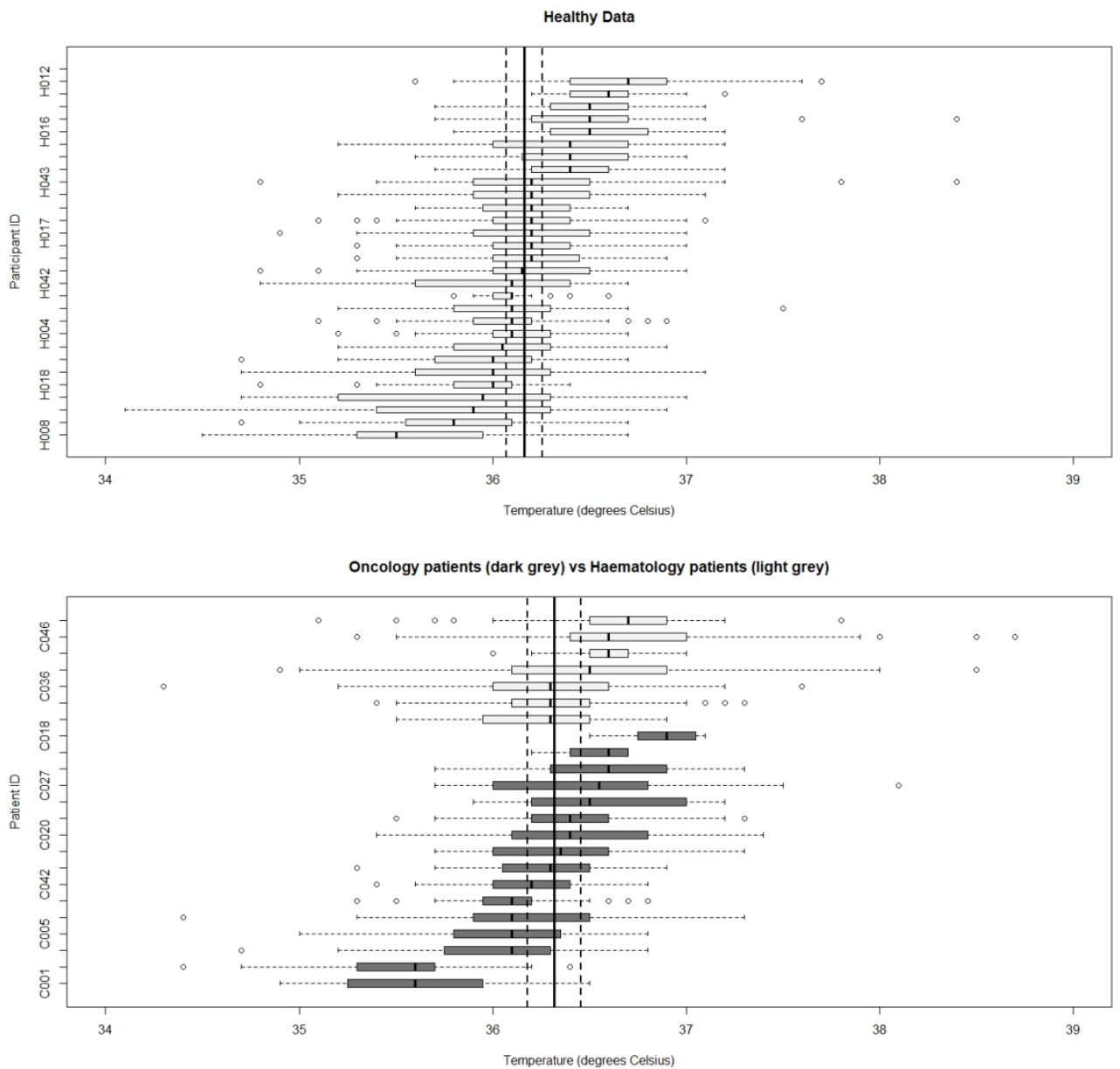

Figure 2 - Temperatures recorded by healthy participants (upper graph), chemotherapy patients (lower graph, haematology (light grey) and oncology (dark grey) patients), ordered by participant mean. For each participant, centre line denotes the median, boxes denote interquartile range; whiskers denote $1.5 x$ the interquartile range above or below the upper and lower quartile respectively; dots are outliers outside of the whisker definition. Solid vertical line denotes the sample mean, with dotted vertical lines representing $95 \%$ confidence intervals. 
Patient C011

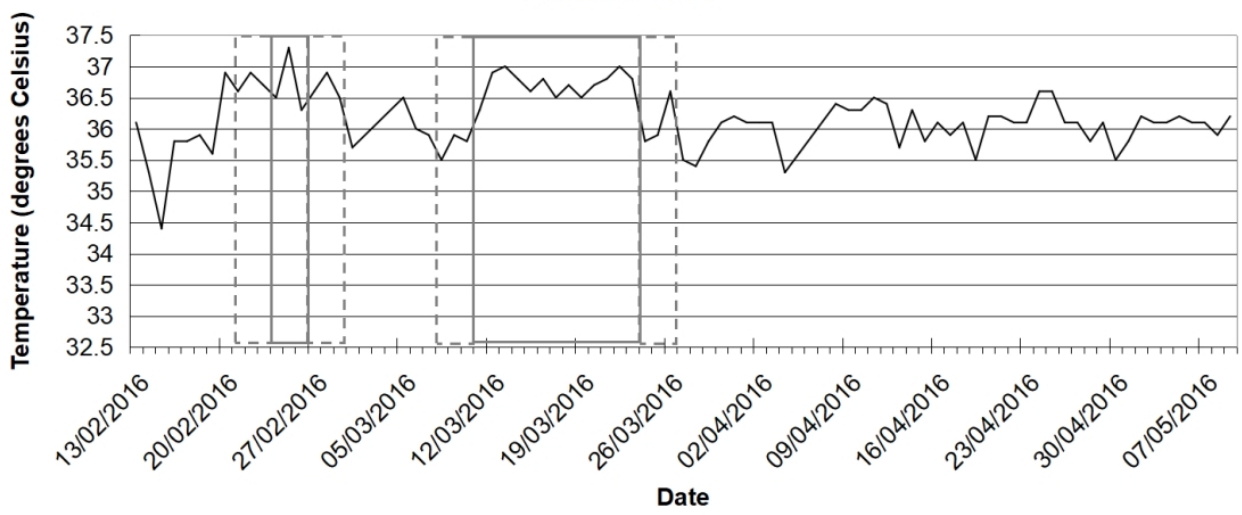

\section{Patient C046}

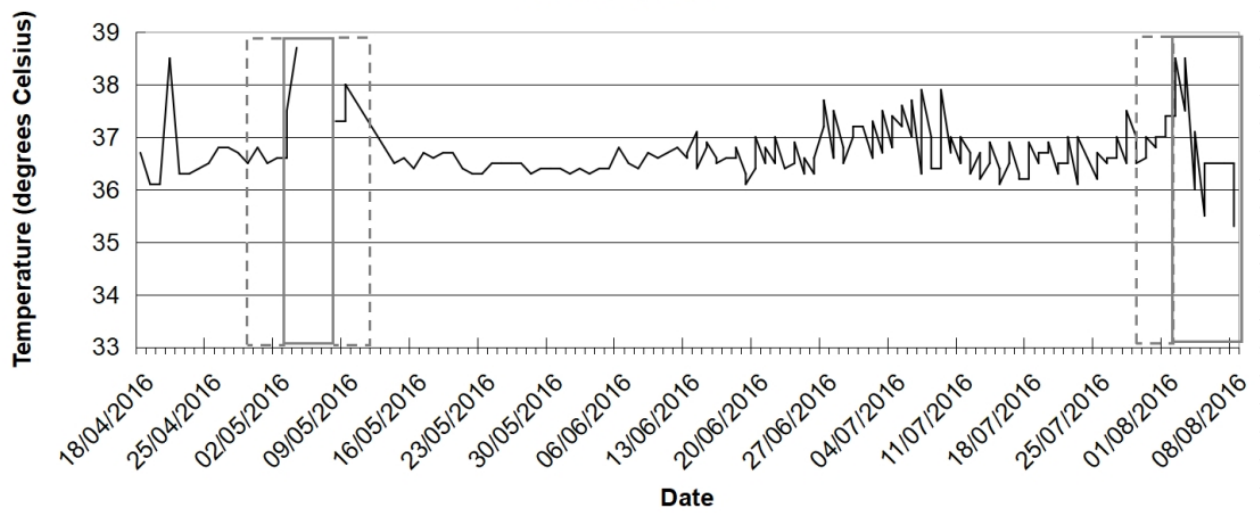

Figure 3 - Individual temperature monitoring graphs for chemotherapy patients who experienced neutropenic sepsis. Solid box indicates the period of the admission for neutropenic sepsis and dashed box three days either side. 